\title{
Efeito da suplementação da dieta com óleo de babaçu sobre a composição do sangue e leite de éguas em lactação
}

Rodolfo Cláudio SPERS ${ }^{1}$

Aleksandrs SPERS²

Wilson Roberto

FERNANDES ${ }^{2}$

José Antônio VISINTIN²

Cledson Augusto GARCIA ${ }^{1}$

Corrspondência para:

RODOLFOCLÁUDIOSPERS

Rua Palmares, 296 - Bloco-04, Apto-32

17501-510-Marília-SP

Recebido para publicação: 03/10/2005 Aprovado para publicação: 10/12/2006

\author{
1 - Faculdade de Ciências Agrárias, Curso de Pós Graduação UNIMAR, \\ Marília - SP \\ 2 - Faculdade de Medicina Veterinária e Zootecnia, USP, São Paulo - SP
}

\section{Resumo}

Foi investigada a suplementação de dietas com óleo de babaçu nos níveis de $(0,4,8$ e 16\%) na composição do sangue e leite de éguas em lactação. Utilizaram-se 16 éguas em lactação, sem raça definida com peso vivo médio de 441,2 kg. As éguas foram suplementadas com $1,5 \%$ do peso vivo com uma mistura composta de quirera de milho, farelo de trigo e farelo de soja com $16 \%$ de proteína bruta. A água e o volumoso constituído de Napier e suplemento mineral, foram oferecidos ad libitum. Colocadas em baias individuais com $4 \times 5 \mathrm{~m}$ as éguas recebiam as dietas tratamento uma vez ao dia.Elas foram alimentadas durante 42 dias. Coletaram-se amostras de $50 \mathrm{ml}$ de sangue para análise de triglicerídeos e colesterol total e $250 \mathrm{ml}$ de leite colhido manualmente para as análises de acidez, teor de gordura e colesterol. O sangue apresentou, ao término do experimento, os seguintes teores: triglicerídeos $\left(24,32,28\right.$ e $\left.25 \mathrm{mg} \mathrm{dl}^{-1}\right)$, colesterol $\left(107,136,126\right.$ e $\left.129 \mathrm{mg} \mathrm{dl}^{-1}\right)$ e para o leite, os teores encontrados foram: (acidez $10,9,8$ e $\left.7^{\circ} \mathrm{D}\right)$, gordura $(0,6 ; 1,1 ; 0,9$ e $0,9 \%)$, colesterol na gordura do leite $\left(366,5 ; 308,6 ; 447,9\right.$ e $\left.491,0 \mathrm{mg} \cdot 100 \mathrm{~g}^{-1}\right)$. A suplementação com óleo de babaçu aumentou os níveis de triglicerídeos e colesterol plasmáticos com efeito linear. Quanto ao leite, diminuiu o teor de acidez e aumentou os teores de gordura com efeito linear, mas não afetou o teor de colesterol.

\section{Introdução}

Os eqüinos, à semelhança dos outros animais, exigem na sua alimentação vários nutrientes como, energia, carboidratos e lipídeos, proteínas e ainda minerais e vitaminas. Esses nutrientes atendem às necessidades das diferentes fases da vida animal: manutenção, crescimento, engorda, gestação, lactação e trabalho. De todas as fases citadas, a lactação é a mais exigente, pois apresenta a maior demanda de nutrientes, principalmente de energéticos. A égua, nas primeiras semanas de lactação, além dos nutrientes para a manutenção, pode requerer vários outros considerando a possibilidade de estar em nova gestação, de ser submetida a trabalho, ainda que moderado, e de produzir grandes quantidades de leite, cujo principal substrato energético é a lactose. Mas, para atender a toda essa exigência, deve ser nutricionalmente muito bem manejada. Isso, porém, não ocorre, pois em geral associa-se a produção de leite apenas com a produção comercial da vaca leiteira, não se levando em conta que todos os mamíferos apresentam tal capacidade. Apesar de a vaca ser especialista em produzir grande quantidade de leite por um período mais longo que outras fêmeas, a égua pode produzir até $24 \mathrm{~kg}$ de leite por dia. Uma das falhas mais comuns cometidas na criação de eqüinos, tanto pelos proprietários como pelos próprios nutricionistas, é a de sub-alimentar as fêmeas durante a lactação. Torna-se indispensável, 
portanto corrigir esse descuido e atender bem às suas necessidades através dos alimentos e seus nutrientes, a começar pelo provimento energético ${ }^{1}$. Afinal, evitando-se a subalimentação e de seu potro, desvia-se dos problemas envolvidos, como crescimento da égua reduzido, baixa eficiência reprodutiva e predisposição a várias enfermidades como assegura Martin, Mcmeniam e Dowset ${ }^{2}$.

Entretanto a manutenção e as condições de produção da lactante e do potro não são suas únicas necessidades a serem atendidas através das fontes energéticas. No entanto, poucos estudos foram realizados a respeito das diferentes fontes de suplementação energética para éguas na fase de lactação $\mathrm{O}^{3,4}$ estudaram a suplementação com o carboidrato (amido) um nutriente energético proveniente principalmente de cereais, como o milho e a aveia. Mais escassos são os trabalhos em que a suplementação energética é feita com gordura, como na avaliação de Zimmerman ${ }^{5}$, que não encontrou modificações nos teores de gordura e proteína, nem na quantidade de leite produzida. Contrariamente, Davinson et al. ${ }^{6}$ observaram que, quando se aumenta a gordura na dieta, obtém-se o aumento dos seus teores no leite. Diante da escassez de pesquisas sobre o tema, Doreau e Boulot ${ }^{7}$, em revisão sobre a produção de leite de éguas, confirmaram a necessidade de mais trabalhos nessa área. A literatura mais recente tem apresentado trabalhos com a suplementação de gordura, mas para animais de esporte. Assim, a partir da última década, as gorduras receberam maior atenção quando comparadas com outros nutrientes acrescentados à alimentação animal, em virtude de sua grande importância na qualidade das dietas. Elas oferecem as seguintes vantagens: aumentam a aceitabilidade das rações: proporcionam maior densidade energética, constituem fontes de ácidos graxos essenciais; são importantes na absorção das vitaminas lipossolúveis; diminuem a poeira das rações e ainda estabilizam a mistura de alimentos.
Dessa forma, com a perspectiva do potencial de aproveitamento e consumo, desenvolveramse diferentes tipos de gordura, algumas de alta qualidade e presentes no mercado a preços competitivos. Entre as gorduras naturais disponíveis, o óleo de babaçu se destaca por apresentar grande quantidade de ácidos graxos de cadeia carbônica curta e média (C8:0 e C12:0), enquanto em outras gorduras vegetais, como a da soja e amendoim, predominam os ácidos graxos de cadeia longa (C18:0 e C18:1).

Os resultados obtidos com o uso do óleo de babaçu na dieta animal não podem ser extrapolados de animais de outras espécies. Aplicam-se somente ao eqüino, animal herbívoro monogástrico que dispõe de mecanismos específicos reguladores de sua ingestão e secreções digestivas ${ }^{8}$.

As gorduras saturadas não são, porém, uma exclusividade dos produtos animais, podendo ser encontradas também em alguns vegetais - no óleo de coco de babaçu, no azeite-de-dendê e no chocolate? Manzano, Esteves e Wanderley ${ }^{10}$, estudaram os efeitos da suplementação de gorduras animal e vegetal nas concentrações de colesterol sangüíneo de eqüinos em crescimento. Verificaram uma diferença significativa $(\mathrm{P}<0,05)$, na suplementação energética, quando então o colesterol alcançou concentrações de 110,10 e 109,19 mg.dl ${ }^{-1}$, respectivamente, para as gorduras animal e vegetal, em comparação a 89,6 $\mathrm{mg} \cdot \mathrm{ml}^{-1}$ para animais não suplementados.

Segundo Weaver ${ }^{11}$, de todos os mamíferos, os eqüinos são os que têm o leite mais semelhante ao dos primatas, pelo fato de ele apresentar na sua composição mais carboidratos do que proteína e gordura. Com o avanço da lactação, observam-se alterações na composição desse leite, com a diminuição da proteína, acompanhada pelo aumento da lactose e da quantidade consumida pelo potro, de maneira que a ingestão de energia bruta tende a satisfazer as exigências nos primeiros meses de lactação.

A composição do leite das éguas é discutida por vários pesquisadores como 
Neseni et al. ${ }^{12}$ e Neuhaus ${ }^{13}$, revelando que o conteúdo de gordura pode variar de 0 a $7 \%$. Em trabalho de revisão, Cherepanova e Belokobylenko ${ }^{14}$ apresentam os seguintes valores: 0,$15 ; 0,5$ a 1,$1 ; 1,1$ a 1,4 e 3,5 a 7,2\% respectivamente para os leites cisternal, alveolar, leite forçado com ocitocina e residual. No que tange ao uso de ocitocina, é necessário ter cautela, pois Doreau et al. ${ }^{1.5}$ e Smolders et al. ${ }^{16}$, verificaram o aumento dos teores de gordura do leite quando ela foi utilizada, embora não se tenham observado variações dos teores de carboidratos e proteína bruta. Também analisando a concentração de gordura no leite equino Fedotov e Akimbekov ${ }^{17}$, encontraram valor médio de $1,59 \%$. Registraram ainda, concentração de 1,25\% para o colostro, a qual aumentou para $2,5 \%$ após 12 horas e reduziu para 1,82\% no décimo dia, dados também revelado pelo trabalho de Csapo et al. ${ }^{18}$.Com o mesmo enfoque Doreau et al. ${ }^{19}$, Kulisa ${ }^{20}$, Oftedal e Hintz ${ }^{21}$, Smolders et al. ${ }^{16}$, Storch ${ }^{22}$, registraram teores de gordura que variaram de 1,05 a 1,29\% em média, o que representa $50,6 \mathrm{kcal}^{1} 100 \mathrm{~g}^{-1}$ de leite. Ainda com relação à gordura e energia bruta do leite, alguns autores Doreau et al. ${ }^{23}$ Pagan e Hintz ${ }^{24}$; e Smolders et al. ${ }^{16}$ constataram diminuição dos teores de gordura com o aumento da ingestão de energia sob a forma de carboidratos. Muitas discordâncias podem ser encontradas na literatura, como a média obtida no trabalho de Cabrera, Fernandes e Moraes $^{25}$, que foi de $0,53 \%$ em todo o período experimental. Valor similar $(0,55 \%)$ foi constatado por outros autores, como Inchausti ${ }^{26}$, além de Aschraft; Tyznik ${ }^{27}$ e Linton $^{28}$, que utilizaram outras raças: shetland e quarto-de-milha Forsyth, Rossdale e Thomas $^{29}$ e Gibbs et al. ${ }^{30}$, encontraram resultados mais elevados. Esses últimos afirmam que o decréscimo de gordura no leite é linear durante a lactação, enquanto Ullrey et al. ${ }^{31}$, observaram redução quadrática. Verificando a composição em gordura do leite de éguas da raça purosangue-inglês (PSI), Cabrera, Fernandes e
Moraes $^{25}$ obtiveram análise de regressão, com efeito quadrático: a concentração decrescia após o sétimo dia, chegando a um nível mais baixo $(0,36 \%)$ aos 91 dias.

Formulando dietas em que o consumo diário de energia alternava de 32,15 a 38,81 Mcal.dia, Raut, Ranjhan e Pathak ${ }^{32}$, observaram que, com dietas variando em concentração de carboidratos, houve pequena oscilação dos teores de gordura: de 0,9 a 1,0\% . Contrariamente, Rajic e Sevkovic $^{33}$, constataram aumento de gordura em 104\% durante os três primeiros meses de lactação. São poucos os trabalhos, em que se verificam alterações na composição do leite de éguas em resposta à suplementação com gordura. Davison et al. ${ }^{34}$, concluíram que, após a suplementação, os teores de gordura do leite aumentam ( $\mathrm{P}$ $<0,01)$, por exemplo, de $0,43 \%$ no dia 10 e $0,99 \%$ no dia 60 .

As amplas divergências dos teores de gordura do leite de éguas são atribuídas aos diferentes componentes dietéticos, aos métodos de amostragem empregados nos trabalhos, ao estado fisiológico e às condições nutricionais (Doreau e Boulot) ${ }^{7}$. No que diz respeito às concentrações de ácidos graxos, segundo Doreau e Boulot ${ }^{35}$, o leite de éguas quando comparado com outras espécies de monogástricos contém relativamente altas concentrações (20-35\%) de ácidos graxos, com menos de dezesseis carbonos, mas é pobre em ácido esteárico menos que $2 \%$. Com respeito aos ácidos linoleico e linolênico, eles representaram $32,67 \%$ dos componentes da gordura, superando em mais de duas vezes o valor encontrado no leite da espécie humana. Para Doreau e Boulot ${ }^{35}$, essa particularidade do leite de éguas tem explicação no fato de não ocorrer a hidrogenação dos ácidos graxos insaturados no trato digestivo desses animais antes da absorção. Além disso, os eqüinos são bastante seletivos na escolha do consumo de forragens.

Estudos ressaltam que a presença de ácidos graxos no leite é reflexo dos ácidos graxos presentes na corrente sangüínea 
Ronneberg, Holmer e Lambertsen ${ }^{36}$. Assim espera-se que éguas suplementadas com gorduras ricas em ácidos graxos de cadeia curta e média (óleo de babaçu), apresentem tais substâncias na composição do leite.

\section{Material e Métodos}

Foram utilizadas dezesseis éguas em início de lactação, com potro ao pé e obtidas de gestações com sincronização dos cios e, portanto nascidas de partos ocorridos em períodos próximos. Eram animais sem raça definida com peso médio de 441,2 kg e idade variando de quatro a oito anos. As éguas foram mantidas em pastagem tendo acesso a água e mistura mineral ad libitum $\mathrm{e}$ sendo recolhidas uma vez ao dia em baias individuais para receberem a suplementação alimentar em cochos. A suplementação com o concentrado foi calculada em $1,5 \%$ do peso vivo em matéria seca (Tabelas 1 e 2). Toda e qualquer eventual sobra de ração foi pesada diariamente para o cálculo do consumo efetivo dos nutrientes. Para a determinação da composição do sangue das éguas, coletaram-se amostras de $50 \mathrm{ml}$ de sangue. As amostras foram colhidas pela punção da veia jugular em frasco com tubo à vácuo, com intervalo de 21 dias, ou seja, D-0, D-21 e D-42 . Dessoradas e armazenadas a $-4^{\circ} \mathrm{C}$, no momento oportuno elas foram descongeladas para a separação de alíquotas destinadas às respectivas análises de triglicerídeos e colesterol total.

\section{Composição do leite das éguas:}

Amostras de $250 \mathrm{ml}$ de leite foram coletadas manualmente para as análises em todas as lactantes, com intervalo de 21 dias, sendo: D-0, D-21 e D-42. As coletas realizaram-se durante a suplementação nas baias, sem a presença dos potros. Tomouse cuidado para que a separação da égua de seu potro se desse num período próximo dos intervalos naturais entre as mamadas. $\mathrm{O}$ leite era coletado dos tetos ao acaso, durante 10 minutos e em frasco previamente esterilizado, desprezando-se os primeiros jatos correspondentes ao leite alveolar. As amostras de cada égua foram congeladas e armazenadas a $-4^{\circ}$ C. No momento oportuno, foram descongeladas, tomandose alíquotas para as respectivas análises de acidez, teor de gordura e colesterol. A acidez do leite foi obtida pela titulação ácida, pelo sistema de Graus Dornic, e o teor de gordura, pelo método de Gerber. No que diz respeito á determinação do colesterol, apenas a amostra D-42 foi utilizada, sendo empregados o método de saponificação segundo Kinsella et al. ${ }^{37}$, e a análise cromatográfica.

\section{Delineamento experimental}

O delineamento experimental foi o de blocos ao acaso com quatro tratamentos ( $0,4,8$ e $16 \%$ de óleo de babaçu) e quatro repetições. As exigências nutricionais dos animais foram calculados com base no $\mathrm{NRC}^{38}$. Os resultados foram submetidos à análise de variância e regressão, e as médias dos tratamentos, comparadas pelo teste de Tukey $(\mathrm{P}<0,05)$ e de Steel e Torrie ${ }^{39}$. Utilizou-se o PROC GLM do SAS ${ }^{40}$, sendo adotado o seguinte modelo matemático:

$$
\begin{aligned}
& \mathrm{Y}_{\mathrm{ijk}}=\mathrm{m}+\mathrm{OB}_{\mathrm{i}}+\mathrm{B}_{\mathrm{j}}+\mathrm{e}_{\mathrm{ijk}} \\
& \mathrm{Y}_{\mathrm{ijk}}=\text { observação individual; } \\
& \mathrm{m}^{\mathrm{j}}=\text { média geral; } \\
& \mathrm{OB}^{\mathrm{i}}=\text { efeito do } \mathrm{i} \text { ésimo nível de óleo } \\
& \mathrm{B}_{\mathrm{j}}=\text { efeito do } \mathrm{j} \text { ésimo bloco }(\mathrm{j}=1,2, \\
& \mathrm{e}_{{ }_{\mathrm{ij} \mathrm{j}}}=\text { erro experimental. }
\end{aligned}
$$$$
\text { de babaçu (i = 1, 2, } 3 \text { e 4); }
$$$$
3 \text { e } 4) \text {; }
$$

\section{Resultados e Discussão}

A concentração dos níveis plasmáticos de triglicerídeos é apresentada na tabela 3 . Ao serem submetidos à análise de variância, esses revelaram efeito significativo $(\mathrm{P}<0,01)$ para os tratamentos realizados nos dias D21 e D-42. Pelo teste de Tukey, no D-21 a maior concentração de triglicerídeos se deu no tratamento 4-OB, o qual foi diferente estatisticamente $(\mathrm{P}<0,05)$. No período $\mathrm{D}$ 42 , o tratamento 4-OB continuou a 
Tabela 1 - Composição química (\%) dos ingredientes das dietas de lactação

\begin{tabular}{lccccc}
\hline Ingredientes & MS & ED & EE & CHO & PB \\
\hline Quirera de milho & 87,5 & 3,41 & 3,9 & 74,5 & 8,9 \\
Farelo de soja & 90,0 & 2,98 & 1,1 & 40,8 & 45,3 \\
Farelo de trigo & 86,6 & 2,62 & 0,8 & 56,6 & 16,2 \\
Óleo de babaçu & 99,0 & 8,54 & 99,0 & - & - \\
\hline
\end{tabular}

$\mathrm{MS}=\%$ matéria seca; $\mathrm{ED}=$ energia digestível $\left(\mathrm{Mcal}_{\mathrm{kg}}{ }^{1}\right) ; \mathrm{EE}=\%$ extrato etéreo; $\mathrm{CHO}=\%$ carboidratos solúveis; $\mathrm{PB}=\%$ proteína bruta

Tabela 2 - Composição centesimal e calculada da dieta de lactação e seus nutrientes, em função dos níveis 0, 4, 8 e 16 (\%) de suplementação com óleo de babaçu

\begin{tabular}{|c|c|c|c|c|}
\hline \multirow{2}{*}{$\begin{array}{c}\text { Composição (\%) } \\
\text { Ingredientes }\end{array}$} & \multicolumn{4}{|c|}{ Níveis de Óleo de Babaçu } \\
\hline & 0 & 4 & 8 & 16 \\
\hline Quirera de milho & 55,0 & 51,0 & 47,0 & 43,0 \\
\hline Farelo de soja & 20,0 & 20,0 & 20,0 & 20,0 \\
\hline Farelo de trigo & 20,0 & 20,0 & 20,0 & 20,0 \\
\hline Óleo de babaçu & 0,0 & 4,0 & 8,0 & 16,0 \\
\hline Sal mineral & 1,5 & 1,5 & 1,5 & 1,5 \\
\hline Calcário calcítico & 1,5 & 1,5 & 1,5 & 1,5 \\
\hline Fosfato bicálcico & 1,0 & 1,0 & 1,0 & 1,0 \\
\hline Sal comum & 1,0 & 1,0 & 1,0 & 1,0 \\
\hline Total & 100,0 & 100,0 & 100,0 & 100,0 \\
\hline \multicolumn{5}{|c|}{ Composição Calculada (1) } \\
\hline MS & 88,34 & 88,82 & 89,28 & 93,70 \\
\hline ED & 2,99 & 3,20 & 3,40 & 3,94 \\
\hline $\mathrm{EE}$ & 2,48 & 6,32 & 10,13 & 17,89 \\
\hline $\mathrm{CHO}$ & 60,38 & 57,47 & 54,49 & 51,51 \\
\hline PB & 17,24 & 16,88 & 16,52 & 16,16 \\
\hline
\end{tabular}

(1) Valores calculados com base no NRC-Nutrient Requirements of Horses,(1989).

$\mathrm{MS}=\%$ matéria seca; $\mathrm{ED}=$ energia digestível $\left(\mathrm{Mcal}^{\mathrm{k}} \mathrm{kg}^{-1}\right) ; \mathrm{EE}=\%$ extrato etéreo; $\mathrm{CHO}=\%$ carboidratos solúveis; $\mathrm{PB}=\%$ proteína bruta

apresentar a maior concentração de triglicerídeos, mas essa concentração não se manteve aos 8-OB, que não diferiu dos demais. Foram semelhantes os níveis $0-\mathrm{OB}$ e 16-OB.

A análise de regressão (Tabela 4) revelou-se significativa $(\mathrm{P}<0,001)$, e equação com efeito linear (Figura 1) no período D-42. Assim, na avaliação desses resultados, o aumento dos triglicerídeos plasmáticos, pode ser explicado pelo aumento da formação das frações de lipoproteínas, ricas em triglicérides os quais são ocasionados pela maior fixação de gordura dietética no intestino delgado. Esses resultados discordam, entretanto, do trabalho de outros autores como Watson, Packard e Shepherd ${ }^{41}$ que verificaram, após a parição, uma significativa diminuição da concentração de triglicerídeos por causa da maior atividade da lipase lipoprotéica do tecido mamário, da qual resulta maior síntese de gordura do leite. Vale lembrar, porém, que nesses casos as éguas não receberam nenhuma suplementação dietética, sendo os resultados obtidos com éguas em condição 
de campo, alimentando-se apenas de pastagens. Também Manzano, Esteves e Wanderley ${ }^{10}$, porém com suplementação, submeteram eqüinos em crescimento a quatro níveis crescentes de gordura animal $(0 ; 2,5 ; 5,0$ e $7,5 \%)$ e verificaram que os triglicerídeos séricos não tiveram diferenças significativas $(\mathrm{P}<0,05)$. Os valores iniciais foram 24, 24, 23 e $23 \mathrm{mg}^{-\mathrm{dl}^{-1}}$, e os finais, 24, 32, 28 e $25 \mathrm{mg} \cdot \mathrm{dl}^{-1}$, respectivamente.

Resultados referentes ao colesterol, apresentados na (Tabela 3), revelaram diferenças significativas $(\mathrm{P}<0,05)$ quando se compararam os tratamentos. Os valores iniciais obtidos foram de 107, 107, 104 e 104 mg.dl ${ }^{-1}$, e os finais, de 107, 136, 126 e $129 \mathrm{mg} \cdot \mathrm{dl}^{-1}$. A análise de variância mostrou efeito significativo $(\mathrm{P}<0,03)$ no $\mathrm{D}-21$, com o tratamento 4-OB, estatisticamente $(\mathrm{P}<$ $0,05)$ superior ao $0-\mathrm{OB}$. No D-42, o resultado foi o mesmo, ressalvando que todos os tratamentos com suplementação foram semelhantes entre si e superiores estatisticamente $(\mathrm{P}<0,007)$ ao $0-\mathrm{OB}$. Ao submeterem-se os dados à análise de regressão, persistiu o efeito significativo $(\mathrm{P}$ $<0,001)$, revelando equação com efeito linear (Tabela 4).

Destaca-se que os tratamentos de suplementação com óleo de babaçu, aumentaram tanto os níveis de triglicerídeos quanto os de colesterol com o decorrer do experimento. $\mathrm{O}$ aumento da concentração de colesterol no sangue das éguas pode ser explicado por vários estudos Keys ${ }^{42}$, Ohtani et al. ${ }^{43}$, Watts et $a l .{ }^{44}$, reportaram que a elevação da colesterolemia ocorre em virtude do aumento no consumo de gordura saturada, nesse caso proveniente da suplementação com óleo de babaçu. Assim Knuiman et al. ${ }^{45}$, com humanos, e Watson, Packard e Shepherd ${ }^{41}$ com éguas, verificaram que a concentração de colesterol plasmático é significativamente relacionada com triglicerídeos. Na lactação, de acordo com Worthy et al. ${ }^{46}$, o teor de colesterol permanece alto, e a concentração de ácidos graxos aumenta significativamente. Ao investigarem em ratos, o tempo decorrido da lipemia pós-prandial, quando esses receberam manteiga, óleo de palmeira, de milho e de canola ou azeite, Lay e $\mathrm{Ney}^{47}$ constaram que aqueles que receberam gordura mais saturada mostraram maior concentração de colesterol e, portanto maior lipedemia pósprandial. Por outro lado, a ingestão de óleo de milho insaturado resultou significativamente em menores concentrações de colesterol plasmático. A mesma dedução foi feita por Lichtenstein et al. ${ }^{48}$, mas com humanos. $\mathrm{Na}$ análise da composição do leite das éguas, avaliaram-se a acidez, o teor de gordura, o colesterol no leite e na gordura do leite e o perfil dos ácidos graxos saturados, mono e polissaturados. Segundo Doreau et al. ${ }^{15}$, para se obterem dados precisos sobre a composição do leite de éguas, deve-se dar atenção à metodologia da colheita. As amostras de leite devem ser colhidas no intervalo entre as mamadas do potro para que ocorra a reposição do leite na glândula. No presente estudo, verificou-se que esse tempo é de trinta minutos no início da lactação e de noventa minutos aos dois meses de lactação.

Os resultados referentes à acidez do leite nos dias D-0, D-21 e D-42 encontramse na tabela 5. Tanto na análise de variância, como no teste de Tukey, não houve efeito significativo nos dois períodos iniciais. Esse efeito só foi averiguado D-42 ( $\mathrm{P}<0,01)$. Nos tratamentos com suplementação, os níveis de acidez foram semelhantes entre si, apenas no tratamento $16-\mathrm{OB}$ eles foram menores que no $0-\mathrm{OB}$ no período D-42. Pela análise de regressão, persistiu a significância $(\mathrm{P}<$ 0,008), cuja equação linear é apresentada na tabela 6. A significância com efeito linear nos níveis de suplementação com óleo de babaçu, é demonstrada na figura 2 .

Os resultados com a gordura do leite são também apresentados na tabela 5. A análise de variância revelou, quanto à gordura, diferenças significativas, com exceção do $\mathrm{D}-0$, sendo $(\mathrm{P}<0,005)$ para $\mathrm{D}$ 21 e $(\mathrm{P}<0,007)$ para D-42. Para D-21, o tratamento 4-OB apresentou o maior teor de gordura (1\%), taxa superior em relação a 
Tabela 3 - Concentração de triglicerídeos (TG) e colesterol (COL) no sangue das éguas, com 0 dias (D-0), 21 dias (D-21) e 42 dias (D-42) em função dos níveis (\%) de suplementação com óleo de babaçu

\begin{tabular}{lccccccc}
\hline Variáveis & 0-OB & 4-OB & 8-OB & $16-O B$ & $X$ & CV & $(\mathrm{P}>\mathrm{F})$ \\
\hline TG mg.dl ${ }^{-1}$ & & & & & & & \\
D-0 & 24 & 24 & 23 & 23 & 24 & 11,21 & 0,99 \\
D-21 & $25^{\mathrm{b}}$ & $31^{\mathrm{a}}$ & $25^{\mathrm{b}}$ & $24^{\mathrm{b}}$ & 26 & 10,02 & 0,01 \\
D-42 & $24^{\mathrm{b}}$ & $32^{\mathrm{a}}$ & $28^{\mathrm{ab}}$ & $25^{\mathrm{b}}$ & 27 & 9,42 & 0,01 \\
& & & & & & & \\
COL mg.dl ${ }^{-1}$ & & & & & & & \\
D-0 & 107 & 107 & 104 & 104 & 105 & 6,32 & 0,82 \\
D-21 & $107^{\mathrm{b}}$ & $125^{\mathrm{a}}$ & $119^{\mathrm{ab}}$ & $120^{\mathrm{ab}}$ & 118 & 6,03 & 0,03 \\
D-42 & $107^{\mathrm{b}}$ & $136^{\mathrm{a}}$ & $126^{\mathrm{a}}$ & $129^{\mathrm{a}}$ & 125 & 5,11 & 0,007 \\
\hline
\end{tabular}

$\mathrm{X}=$ media dos níveis de $(\mathrm{OB}) ; \mathrm{CV}=$ coeficiente de variação; $(\mathrm{P}>\mathrm{F})=$ probabilidade \% de significância do teste $\mathrm{F}$.

$\mathrm{a}, \mathrm{b}, \mathrm{c}$ ed $=$ médias com letras diferentes diferem estatisticamente pelo teste de Tukey $(\mathrm{P}<0,05)$

Tabela 4 - Análise de regressão da concentração de triglicerídeos e colesterol no sangue de éguas, com 42 dias (D-42), em função dos níveis (\%) de suplementação com óleo de babaçu

\begin{tabular}{llcccccc}
\hline Variáveis & Equação & $\mathrm{R}^{2}$ & $\mathrm{Pr}>\mathrm{F}$ & $\mathrm{CV}$ & $\mathrm{EPGL}$ & $\mathrm{EPGQ}$ & EPI \\
TG mg.dl $^{-1}$ & $\mathrm{Y}=24+0,5 \mathrm{X}$ & 0,73 & 0,001 & 8,61 & 0,98 & 0,18 & 1,17 \\
COL mg.dl $^{-1}$ & $\mathrm{Y}=107+1,27 \mathrm{X}$ & 0,83 & 0,001 & 4,48 & 2,33 & 0,44 & 2,79 \\
\hline
\end{tabular}

$\mathrm{R}^{2}=$ coeficiente de determinação; $\mathrm{Pr}>\mathrm{F}=$ probabilidade $\%$ de significância do teste $\mathrm{F} ; \mathrm{CV}=$ coeficiente de variação; $\mathrm{EPGL}=$ erro padrão do gradiente linear;EPGQ = Erro padrão do gradiente quadrático;EPI = erro padrão do intercepto

0-OB $(0,6 \%)$ e $8-\mathrm{OB}(0,7 \%)$, mas estatisticamente semelhante ao teor encontrado em $16-\mathrm{OB}(0,8 \%)$, pelo teste de Tukey. Os resultados submetidos à análise de regressão (Tabela 6), mostraram efeito significativo $(\mathrm{P}<0,008)$, considerado linear quando interpretado pela equação de regressão figura 2 .

No D-42, a tendência persistiu com intensidade semelhante $(\mathrm{P}<0,007)$, (Tabela 5). O tratamento com 4-OB apresentou novamente maiores teores de gordura $(1,1 \%)$,índice superior ao observado em $0-\mathrm{OB}(0,6 \%)$ e semelhante estatisticamente ao das demais suplementações: 8-OB e 16-OB, ambas com (0,9\%), segundo, teste de Tukey. Os resultados que passaram por análise de regressão no período D-42 revelaram valores semelhantes entre si $(\mathrm{P}<0,001)$ descritos no período D-42 (Tabela 6 ). A equação de regressão apresentou o mesmo efeito linear (Figura 2). Ressalta-se a tendência linear inversa dos teores de acidez de gordura do leite, ou seja, à medida que aumenta a suplementação com óleo de babaçu, diminui para a acidez e aumenta a gordura. Diante do aumento dos teores de gordura do leite de éguas suplementadas óleo, recomenda-se a adição já preconizada por Davison et al. ${ }^{6,34}$ de $5 \%$ de óleo de milho na dieta das éguas em início de lactação, visando esse aumento.

Os resultados submetidos à análise de variância do colesterol na gordura em função dos níveis (\%) de suplementação com óleo de babaçu, são apresentados na tabela 5 , não houve efeito significativo, o que persistiu na análise de regressão, observada na Tabela 6 .

\section{Conclusões}

Diante dos resultados obtidos, verificouse que a suplementação da dieta de éguas em lactação com óleo de babaçu proporcionou: aumento dos níveis de triglicerídeos e colesterol plasmático, com efeito linear; diminuição do teor de acidez e elevação do teor de gordura do leite, com efeito linear.

Quanto ao teor de colesterol no leite, esse não foi afetado. 


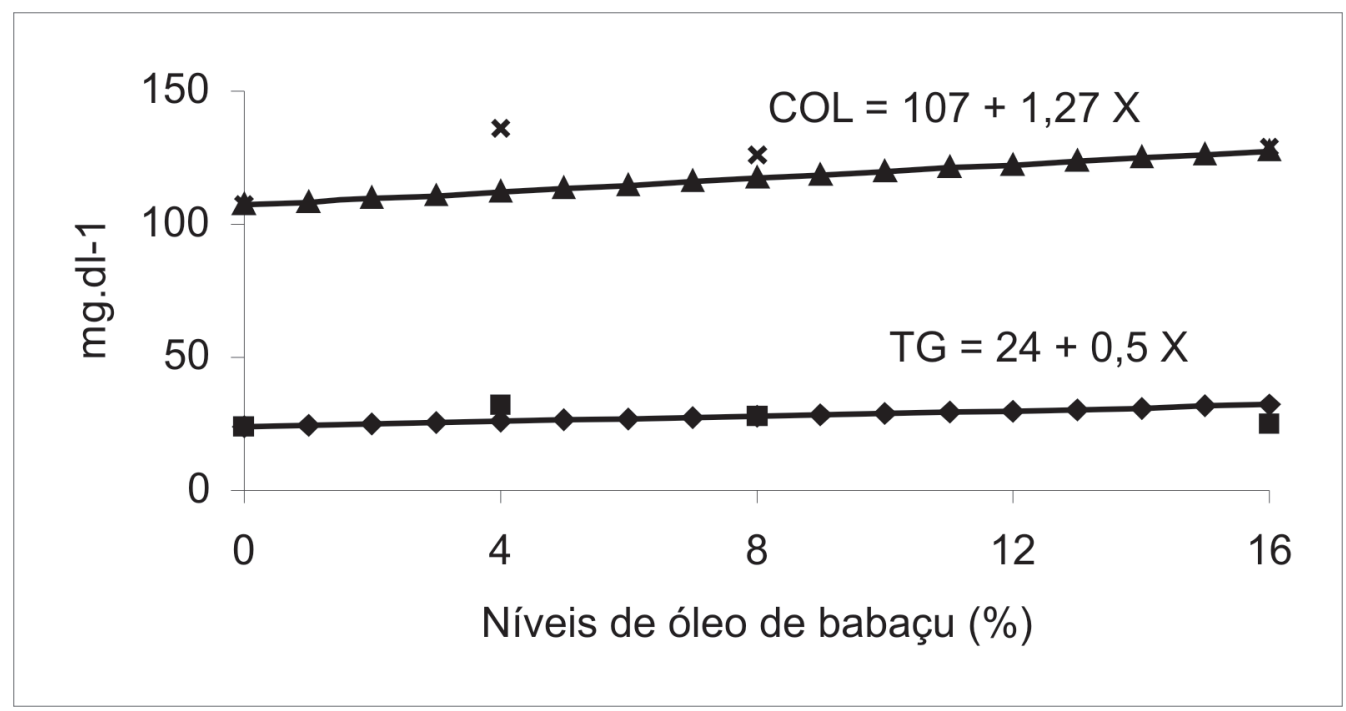

Figura 1 - Equação de regressão da concentração de triglicerídeos e colesterol no sangue de éguas, com 42 dias (D-42), em função dos níveis (\%) de suplementação com óleo de babaçu

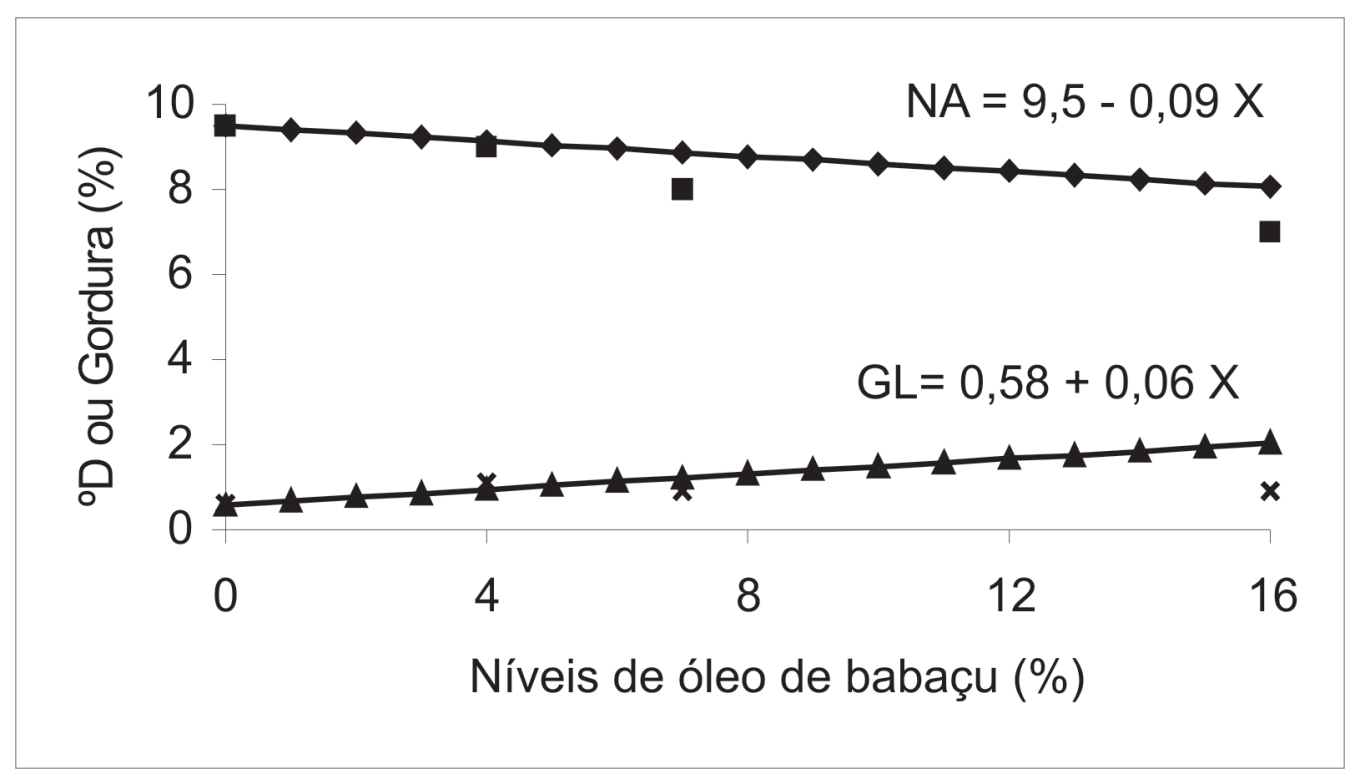

Figura 2- Equação de regressão do Nível de Acidez (NA) e composição (\%) do leite de éguas em Gordura (GL) aos 42 dias (D-42) em função dos níveis (\%) de suplementação com óleo de babaçú 
Tabela 5 - Nível de acidez (NA) e composição (\%) do leite das éguas em gordura e colesterol na gordura com 0 dia (D-0), 21 dias (D-21) e 42 dias (D-42) em função dos percentuais de suplementação com óleo de babaçu

\begin{tabular}{|c|c|c|c|c|c|c|c|}
\hline Variáveis & $0-\mathrm{OB}$ & $4-\mathrm{OB}$ & $8-\mathrm{OB}$ & $16-\mathrm{OB}$ & $x$ & $\mathrm{CV}$ & $(P>F)$ \\
\hline \multicolumn{8}{|l|}{$\mathrm{NA}\left({ }^{\circ} \mathrm{D}\right)$} \\
\hline D-0 & 10 & 8 & 10 & 9 & 9 & 9,53 & 0,07 \\
\hline D-21 & 9 & 9 & 8 & 9 & 9 & 12,62 & 0,65 \\
\hline D-42 & $10^{\mathrm{a}}$ & $9^{a b}$ & $8^{\mathrm{ab}}$ & $7^{\mathrm{b}}$ & 9 & 9,95 & 0,01 \\
\hline \multicolumn{8}{|l|}{$\mathrm{GL},(\%)$} \\
\hline D-0 & 0,6 & 0,7 & 0,6 & 0,6 & 0,6 & 27,72 & 0,83 \\
\hline D-21 & $0,6^{\mathrm{b}}$ & $1,0^{\mathrm{a}}$ & $0,7^{\mathrm{b}}$ & $0,8^{\mathrm{ab}}$ & 0,8 & 14,05 & 0,005 \\
\hline D-42 & $0,6^{\mathrm{b}}$ & $1,1^{\mathrm{a}}$ & $0,9^{\mathrm{ab}}$ & $0,9^{\mathrm{ab}}$ & 0,8 & 17,91 & 0,007 \\
\hline \multicolumn{8}{|l|}{ CGL } \\
\hline $\mathrm{COL}\left(\mathrm{mg} .100 \mathrm{~g}^{-1}\right)$ & 366,5 & 308,6 & 447,9 & 491,0 & 403,5 & 61,40 & 0,73 \\
\hline
\end{tabular}

$\mathrm{X}=$ média dos níveis de óleo de babaçu; $\mathrm{CV}=$ coeficiente de variação; $(\mathrm{P}>\mathrm{F})=$ probabilidade \% de significância do teste $\mathrm{F}$.

$\mathrm{a}, \mathrm{b}, \mathrm{ced}=$ médias com letras diferentes diferem estatisticamente pelo Teste de Tukey $(\mathrm{P}<0,05)$

Tabela 6 - Análise de regressão do Nível de Acidez (NA) e composição (\%) do leite de éguas em Gordura (GL) e Colesterol (COL) na Gordura (CGL) com 0 dias (D-0), 21 dias (D-21) e 42 dias (D-42) em função dos níveis (\%) de suplementação com óleo de babaçú (OB)

\begin{tabular}{|c|c|c|c|c|c|c|c|}
\hline Variáveis & Equação & $\mathrm{R}^{2}$ & $\operatorname{Pr}>\mathrm{F}$ & $\mathrm{CV}$ & EPGL & EPGQ & EPI \\
\hline \multicolumn{8}{|l|}{$\mathrm{NA},\left({ }^{\circ} \mathrm{D}\right)$} \\
\hline D-0 & $Y=9,75-1,08 X+0,21 X^{2}$ & 0,36 & 0,13 & 11,47 & 0,44 & 0,08 & 0,53 \\
\hline D-42 & $Y=9,5-0,09 X$ & 0,61 & 0,008 & 10,05 & 0,35 & 0,07 & 0,42 \\
\hline \multicolumn{8}{|l|}{$\mathrm{GL}(\%)$} \\
\hline D-0 & $Y=0,63+0,06 X-0,01 X^{2}$ & 0,09 & 0,77 & 24,52 & 0,06 & 0,01 & 0,08 \\
\hline D-21 & $Y=0,58+0,22 X-0,04 X^{2}$ & 0,78 & 0,008 & 12,47 & 0,04 & 0,01 & 0,05 \\
\hline D-42 & $Y=0,58+0,06 X$ & 0,71 & 0,001 & 16,32 & 0,06 & 0,01 & 0,07 \\
\hline \multicolumn{8}{|l|}{ CGL } \\
\hline $\mathrm{COL}\left(\mathrm{mg} 100 \mathrm{~g}^{-1}\right)$ & $Y=366,5-56,3 X+12,62 X^{2}$ & 0,11 & 0,68 & 56,24 & 94,9 & 17,9 & 113,5 \\
\hline
\end{tabular}

$\mathrm{R}^{2}=$ coeficiente de determinação; $\mathrm{Pr}>\mathrm{F}=$ probabilidade $\%$ de significância do teste $\mathrm{F} ; \mathrm{CV}=$ coeficiente de variação; $\mathrm{EPGL}=$ erro padrão do gradiente linear; $\mathrm{EPGQ}=$ erro padrão do gradiente quadrático; $\mathrm{EPI}=$ erro padrão do intercepto

\section{Effect of energetic supplementation with coco-nut oil to lactating mares diets on blood and milk composition}

\section{Abstract}

The effect of dietary supplementation with coconut oil (CO) with four levels $(0,4,8$ and $16 \%)$ on the variation of blood and milk composition of lactating mares were investigated. Sixteen lactating mares were used with average live weight of $441.2 \mathrm{~kg}$. Mares were supplemented with $1,5 \%$ of live weight with concentrate composed of corn meal, wheat bran and soybean meal with $16 \%$ crude protein. Forage was Napier grass and mineral supplement and water supplied "ad libitum". Mares were allotted to 16 pens $(4 \times 5 \mathrm{~m})$ and randomly assigned to receive each of four diets once a day during 42 days. Samples of blood $(50 \mathrm{ml})$ were collected for analysis of triglycerides
Key-words:

Mares.

Blood.

Milk.

Triglycerides.

Cholesterol. 
and cholesterol. Samples of milk $(250 \mathrm{ml})$ were collected manually for analyses of acidity, fat and cholesterol. At the end of the experiment the supplementation with $\mathrm{CO}$ showed respectively for blood: triglycerides, 24; 32; 28 and $25 \mathrm{mg} \mathrm{dl}^{-1}$; cholesterol, 107; 136; 126 and $129 \mathrm{mg} \mathrm{dl}^{-1}$ and for milk: acidity, $10 ; 9 ; 8$ and $7^{\circ} \mathrm{D}$; fat, 0,$6 ; 1,1 ; 0,9$ and $0,9 \%$. Cholesterol in the fat of the milk, 366.5; 308.6; 447.9 and 491.0 mg. $100 \mathrm{~g}^{-1}$. The supplementation with CO, increased the triglycerides levels and plasmatic cholesterol, with linear effect; they reduced the level of acidity and increased fat content of milk with linear effect and they had no effect on cholesterol content in the milk.

\section{Referências}

1 CUNHA, T. J. Horse feeding and nutrition. New York, Academic Press, 1991. p. 216-234.

2 MARTIN, R. G.; MCMENIAM, N. P.; DOWSET, K. F. Milk and water intakes of foal sucking grazing mares. Equine Veterinary Journal, v. 24, n. 4, p. 295-299, 1992.

3 SHEPETOV, F. N.; MULIKOV, A. I.; GAIDASH, V. A. Breeding Dairy horses around Stalingrad. Konevodstvo Konnyi Sport., v. 6, p. 41-45, 1953.

4 PAGAN, J. D.; HINTZ, H. F.; ROUNSAVILLE, T. R. The digestible energy requirements of lactating pony mares. Journal of Animal Science, v. 58, n. 6, p. 13821387, 1984.

5 ZIMMERMAN, R. A. Effect of ration on composition of mare's milk. In: EQ. Nutritions Physiol. Symp. 9., Michigan, 1985. Proceedings... [S. I.:s. n.], 1985. p. 96-102.

6 DAVISON, K. E. et al. C. Lactation and reproductive performance of mares fed added dietary fat during late gestation and early lactation. In: EQ. Nutr. Physiol. Symp., 10., 1987, Colorado. Proceedings... Colorado: Colorado State University, 1987, p. 87-92

7 DOREAU, M.; BOULOT, S. Recent knowledge on milk maré production: A Review. Livestock Production Science, v. 22, p. 213-235, 1989a

8 LINZELL, J. L. et al. Mammary and whole-body metabolism of glucose, acetate and palmitate in the lactating horse. Proc. Nutr. Soc., v. 31, p. 72-73, 1972.

9 QUINTÃO, E.; NAKANDAKARE, E. R. Manual de referência em dislipidemias, Novartis. [S. I.: s. n.], 1997.

10 MANZANO, A.; ESTEVES, S. N.; WANDERLEY, R. C. Óleo de soja e gordura animal na alimentação de eqüinos. In: REUNIÃO ANUAL DA SBZ, 31., 1995 Campo Grande, MS. Anais... Campo Grande: Sociedade Brasileira de Zootecnia, 1995. p. 570.

11 WEAVER, L. T. Milk and the neonatal gut: comparative lessons to be learnt. Cambridge and medical Research Council. Equine Veterinary Journal, v. 18, n. 6, p. 427-429, 1986.

12 NESENI, R. et al. Milchleistung und
Milchzusammensetzung von Stuten im Verlaufe der Laktation. Arch.Tierzucht, v. 1, p. 91-129, 1958.

13 NEUHAUS, V. Milch und Milchgewinnung von Pferdestuten. Z.Tierzucht. v. 73, p.59-61, 1959.

14 CHEREPANOVA, V. P.; BELOKOBYLENKO, V. T. Milk ejection characteristics in machine milk of mares. Dairy Sci., n. 37, p. 691, 1974.

15 DOREAU, M. et al. Milking lactating mares using oxytocin: milk volume and composition. Reproduction, Nutrition, Development, v. 26, n. 1A, p. 1-11, 1986.

16 SMOLDERS, E. A. et al. Livestock Production Science, v. 25 n. 1-2, p. 163-171, 1990.

17 FEDOTOV, P.; AKIMBEKOV, B. Increasing milk production of kushum mares. Konevodstvo I Konnyi Sport., n. 11, p. 6-7, 1983.

18 CSAPO, J. et al. Composition oh the milk of the mare, $1^{\text {st }}$ Paper: Fat content and fatty acid composition of the colostrums and milk. Allattenyesztes es Takarmanyozas., v. 42, n. 2, p. 131-146, 1993.

19 DOREAU, M. et al. Can mares be fed on small quantities of concentrates after foaling? Journee de la Recherche Chevaline, n. 14e, p. 103-114, 1988.

20 KULISA, M. Select amino acids, fatty acids and Nacetylneuraminic acid in mare milk. In: ANNUAL MEETING OF THE EUROPEAN ASSOCIATION FOR ANIMAL PRODUCTION. BUDAPEST, HUNGARY, 1986b. Proceedings... [S. I.: s. n.], 1986. p. 310-311.

21 OFTEDAL, O T.; HINTZ, H. F. Lactation in the horse: milk composition and intake by foals. Journal of Nutrition, v. 113, n. 10, p. 2096-2106, 1983.

$22 \mathrm{STORCH}, \mathrm{G}$. Composition and properties of mares milk and koumiss, with particular regard to dietetic aspects. Thesis, Justus-Liebig-Univ., Giessen german Federal Republic, Inst. Fur Tierarztliche Nahrungsmittelkunde. p. 98, 1985.

23 DOREAU, M. et al. Yield and composition of milk from lactating mares : effect of lactating stage and individual differences. Journal of Dairy Research, v. 57, n. 4, p. 449-454, 1990.

24 PAGAN, J. D.; HINTZ, H. F. Composition of milk from pony mares fed various levels of digestible energy. New York State College Vet. Med. Cornell Veterinarian, 
v. 76, n. 2, p. 139-148, 1986.

25 CABRERA, L. C.; FERNANDES, O.; MORAES, C. M. M. Composição de leite de éguas P.S.I, e desenvolvimento de suas crias. A Hora Veterinária, ano 10, n. 55, p. 24-29, 1990.

26 INCHAUSTI, D. Raza pura sangre de carrera. Buenos Aires, El Ateneo, p. 219-244, 1953.

27 ASCHRAFT, A.; TYZNIK, W. J. Effect of diet on volume and composition in mare's milk. J.Anim.Sci., $\mathrm{v}$. 50, n. 1, p. 248, 1976.

28 LINTON, R. G. The composition of mare's milk. J. Dairy Sci., v. 8, p. 143-172, 1937.

29 FORSYTH, I. A.; ROSSDALE, P. D.; THOMAS, C. R. Studies in milk composition and lactogenic hormones in the mare. J. Reprod. Fert., v. 23, p. 631-635, 1975. Supplement.

30 GIBBS, P. G. et al. Milk production of quarter horses mares during 150 days of lactation. Journal of Animal Science, v. 54, p. 496-499, 1982.

31 ULLREY, D. E. et al. Composition of mare's milk; Journal of Animal Science, v. 25, p. 217-222, 1966.

32 RAUT, B.; RANJHAN, S. K.; PATHAK, N. N. A note on effect of different levels of concentrate feeding with good quality fodders on the yield and composition of milk of Mountain Artillery (MA) mares. Indian Journal of Dairy Science. v. 41, p. 493-495, 1988.

33 RAJIC, I.; SEVKOVIC, N. The nutrition of lactation mares. Veterinarski fakultet, Belgrade, Yugoslavia. Veterinarsk-Glasnik, v. 39, n. 8, p. 899-904, 1985.

34 DAVISON, K. E. et al. Lactation and reproductive performance of mares fed added dietary fat during late gestation and early lactation. Journal of Equine Veterinary Science, v. 11 n. 2, p. 111-115, 1991.

35 DOREAU, M.; BOULOT, S, Methods of measurement of milk yield and composition in nursing mares: A Review. Le Lait, v. 69, p. 159-171, 1989 b.

36 RONNEBERG, R.; HOLMER, G.; LAMBERTSEN, G. Comparative Metabolism of erucic and oleic acid in hepatocytes from rats fed partially hydrogenated marine oil or palm oil. Animal Nutrition Metabolism, v. 31, n. 3, p. 160-169, 1987.

37 KINSELLA, J. E. et al. Método de Saponifacação. Journal of food Biochemistry, v. 23, n. 1 p. 131-140, 1977.

38 NRC. Nutrient requirements of horses. 5ed. Washington D. C.: N.R.C., 1989. p. 100.

39 STEEL, R. G. D.; TORRIE, J. H. Principles and procedures of statistics, $2^{\text {nd }}$ ed. New York: MacgrawHill, 1980, p. 173-177.

40 SAS. User's guide: statistics version 5. Cary, SAS Institute, NC: 1985.
41 WATSON, T. D. G.; PACKARD, C. J.; SHEPHERD, J. Plasma lipid transport in the horse (equus caballus). Comparative Biochemistry and Physiology, v. 106, n. 1, p. 27-34, 1993b.

$42 \mathrm{KEYS}, \mathrm{A}$. Serum cholesterol response to dietary cholesterol. J. Clin. Nutr., v. 40, p. 351, 1984.

43 OHTANI, H. et al. Effects of dietary cholesterol and fatty acids on plasma cholesterol level and hepatic lipoprotein metabolism. J. Lipid Res., v. 31, p. 1413, 1990.

44 WATTS, G. F. et al. Nutrient intake and progression of coronary artery disease. Am. J. Cardiol., v. 73, p. $328,1994$.

45 KNUIMAN, J. T. et al. The cholesterol and high density lipoprotein cholesterol levels in populations differing in fat and carbohydrate intake. Arterioscl. Tromb., v. 7, p. 612, 1987.

46 WORTHY, K. et al. Plasma prolactin concentration and cyclic activity in pony mares during parturition and early lactation. University of Glasgow Veterinary School, Journal of Reproduction and Fertility. v. 77, n. 2, p.569-574, 1986.

47 LAY, H. C.; NEY, D. M. Corn Oil, palm oil and butterfat fractions affect postprandial lipemia and lipoprotein lipase in meal-fed rats. Journal of Nutrition, v. 135, n. 6 , p. $1536-1545,1995$.

48 LICHTENSTEIN, A. H. et al. Effects of canola, corn, and olive oils on fasting and posprandial plasma lipoproteins in humans as part of a national cholesterol education program step 2 diet. Arterioscl. Tromb., v. 13, p. 1533, 1993. 